\title{
CONSTRAINED EXTREMAL PROBLEMS FOR CLASSES OF MEROMORPHIC FUNCTIONS
}

\author{
BY J. A. PFALTZGRAFF AND BERNARD PINCHUK \\ Communicated by Dana S. Scott, October 25, 1968
}

1. Introduction. Let $S(g)$ denote the class of functions $S(z)$ analytic in $D=\{z:|z|<1\}$ which have the integral representation

$$
S(z)=\int_{0}^{2 \pi} g(z, t) d m(t)
$$

where $m(t)$ is a nondecreasing function on $0 \leqq t \leqq 2 \pi, \int_{0}^{2 \pi} d m(t)=1$ and $g(z, t)$ is given for the class. We shall assume that $g(z, t)$ and $g_{t}(z, t)$ are analytic functions of $z$ in $D$ and Lipschitz continuous with respect to $t$ (uniformly for $z$ in a compact subset of $D$ ). G. M. Goluzin has developed a variational method for $S(g)$ [5] which has proved to be useful in the study of extremal problems within various classes of analytic functions $[4],[5],[9],[10]$.

In this note we present a generalization of the Goluzin variational method. The generalization enables one to preserve side conditions imposed on the functions $m(t)$ in (1). Our work was motivated by the fact that various classes of meromorphic functions have structural formulas based on (1) where $m(t)$ must satisfy the additional condition $\int_{0}^{2 \pi} e^{-i t} d m(t)=0$.

Complete proofs and applications of our results will be published elsewhere [8].

2. Constrained variations. Let $A,(\nu=1, \cdots, n)$ be real numbers and let $u_{\nu}(t)(\nu=1, \cdots, n)$ be real valued $C^{1}$ functions on $0 \leqq t \leqq 2 \pi$. The class $\mathrm{S}(g, A)$ is defined to be the class of functions (1) in $\mathrm{S}(g)$ that satisfy the constraints

$$
\int_{0}^{2 \pi} u_{\nu}(t) d m(t)=A_{\nu}, \quad \nu=1, \cdots, n
$$

Let $J\left(T_{1}, \cdots, T_{n}\right)$ denote the determinant of the matrix $\left[u_{k}^{\prime}\left(T_{j}\right)\right]$ $(j, k=1, \cdots, n)$ and let $J_{\nu}=J\left(T_{1}, \cdots, T_{\nu-1}, T, T_{\nu+1}, \cdots, T_{n}\right)$ $(\nu=1, \cdots, n)$ where $T, T_{1}, \cdots, T_{n} \in[0,2 \pi]$. A set of points $T, T_{1}, \cdots, T_{n}$ in $[0,2 \pi]$ is said to be admissible for $m(t)$ if $m(t)$ is not constant in any neighborhood of each of these points and if the determinants $J\left(T_{1}, \cdots, T_{n}\right), J_{\nu}(\nu=1, \cdots, n)$ are all nonzero. 
TheOREM 1. Let $S(z)=\int_{0}^{2 \pi} g(z, t) d m(t)$ belong to $S(g, A)$ and let $T, T_{1}, \cdots, T_{n}$ be an admissible set of points for $m(t)$. Then there exist complex numbers $\lambda_{\nu}(\nu=1, \cdots, n)$ and open intervals $e$ about $T$ such that the functions

$$
S^{*}(z)=S(z)+\epsilon \int_{0}\left\{g_{t}(z, t)+\sum_{\nu=1}^{n} \lambda_{\nu} u_{\nu}^{\prime}(t)\right\}|m(t)-c| d t+O\left(\epsilon^{2}\right)
$$

belong to $\$(g, A)$ for all sufficiently small real $\epsilon$. Furthermore, if $m(t)$ has discontinuities at $t_{1}$ and $t_{2}$ then for all sufficiently small real $\epsilon$ the functions

$$
S^{* *}(z)=S(z)+\epsilon\left(H\left(z, t_{1}\right)-H\left(z, t_{2}\right)\right)+O\left(\epsilon^{2}\right)
$$

belong to $\mathrm{S}(\mathrm{g}, A)$ where

$$
H(z, t)=g(z, t)+\sum_{v=1}^{n} \lambda_{\nu} u_{v}(t) .
$$

REMARK. In all of our applications it is easy to find admissible sets of points for the function $m(t)$ if it is not a step function. Furthermore, any exceptional cases due to step functions can be disposed of by methods of elementary calculus.

3. Extremal problems for meromorphic functions. Let MC denote the class of functions

$$
f(z)=1 / 2+a_{0}+a_{1} z+\cdots, \quad 0<|z|<1,
$$

which are regular in $0<|z|<1$ and which map $D$ conformally onto the exterior of a convex set. The class MC is completely characterized by the structural formula

$$
f^{\prime}(z)=-\frac{1}{z^{2}} \exp \left\{2 \int_{0}^{2 \pi} \log \left(1-z e^{-i t}\right) d m(t)\right\}
$$

where $m(t)$ ranges over the set of nondecreasing functions on $0 \leqq t \leqq 2 \pi$ which satisfy

$$
\int_{0}^{2 \pi} d m(t)=1 \text { and } \int_{0}^{2 \pi} e^{-i t} d m(t)=0 \quad \text { (c.f. [11]). }
$$

ThEOREM 2. Let $F\left(X_{1}, \cdots, X_{n+1}\right)$ be an analytic function from $C^{n}$ into $C$. The functional $J\left(f^{\prime}\right)=\operatorname{Re} F\left(f^{\prime}(z), \cdots, f^{(n)}(z), z\right)(f \in M C$ and $z \neq 0$ a fixed point in $D)$ attains its maximum and minimum over the class MC only for functions of the form

$$
f^{\prime}(z)=-\frac{1}{z^{2}} \prod_{j=1}^{N}\left(1-\epsilon_{j z}\right)^{2 m_{j}}
$$


where $N \leqq n+1,\left|\epsilon_{j}\right|=1, m_{j} \geqq 0(j=1, \cdots, N), \sum_{j=1}^{N} m_{j}=1$ and $\sum_{j=1}^{N} m_{j} \epsilon_{j}=0$.

If $n=1$ in Theorem 2 then the conditions $\sum_{j=1}^{N} m_{j}=1, \sum_{j=1}^{N} m_{j} \epsilon_{j}$ $=0,\left|\epsilon_{j}\right|=1$ and $N \leqq 2$ imply that $m_{1}=m_{2}=1 / 2$ and $\epsilon_{2}=\epsilon_{1}$. Thus the functionals $J\left(f^{\prime}\right)=\operatorname{Re} F\left(f^{\prime}(z)\right)$ attain extreme values over MC for the functions

$$
f(z)=1 / z+a_{0}+a_{1} z, \quad\left|a_{1}\right|=1 .
$$

In particular we have the following distortion and rotation theorem.

TheOREM 3. For $f(z) \in M C$ and $0<|z|<1$

$$
\begin{gathered}
\left|f^{\prime}(z)+1 / z^{2}\right| \leqq 1, \\
\left(1-|z|^{2}\right) /|z|^{2} \leqq\left|f^{\prime}(z)\right| \leqq\left(1+|z|^{2}\right) /|z|^{2},
\end{gathered}
$$

and

$$
\left|\arg f^{\prime}\left(r e^{i \theta}\right)\right| \leqq\left|\pi-2 \theta+\arcsin r^{2}\right|,
$$

in the slit disk $-\pi<\theta<\pi, 0<r<1$. These bounds are all sharp.

Similar results for convex meromorphic functions in $|z|>1$ are known, c.f. [12].

Let $\mathrm{MC}(p), 0<p<1$, denote the class of univalent functions $f(z)$ which are regular in $D$ except for a pole at $z=p$, normalized to satisfy $f(0)=0, f^{\prime}(0)=1$, and which map $D$ onto the exterior of a convex set. This class of functions is completely characterized by the structural formula

$$
f^{\prime}(z)=\frac{p^{2}}{(z-p)^{2}(1-p z)^{2}} \exp \left\{2 \int_{0}^{2 \pi} \log \left(1-z e^{-i t}\right) d m(t)\right\}
$$

where $m(t)$ ranges over the set of nondecreasing functions on $0 \leqq t \leqq 2 \pi$ which satisfy

$$
\int_{0}^{2 \pi} d m(t)=1 \text { and } \int_{0}^{2 \pi}\left(p-e^{-i t}\right) /\left(1-p e^{-i t}\right) d m(t)=0 .
$$

Theorem 4. The functional $J\left(f^{\prime}\right)=\operatorname{Re} F\left(f^{\prime}(z), \cdots, f^{(n)}(z), z\right)$ $(z \neq p, z \in D)$ attains its maximum and minimum over the class $\mathrm{MC}(p)$ only for functions of the form

$$
f^{\prime}(z)=\frac{p^{2}}{(z-p)^{2}(1-p z)^{2}} \prod_{j=1}^{N}\left(1-\epsilon_{j} z\right)^{2 m_{j}}
$$

where $N \leqq n+2,\left|\epsilon_{j}\right|=1, m_{j} \geqq 0(j=1, \cdots, N), \sum_{j=1}^{N} m_{j}=1$ and $\sum_{j=1}^{N} m_{j}\left(p-\epsilon_{j}\right) /\left(1-p \epsilon_{j}\right)=0$. 
The conclusion $N \leqq n+2$ is weaker than the corresponding $N$ $\leqq n+1$ in Theorem 2 . This is due to the more complicated nature of the constraint in $\mathrm{MC}(p)$, cf. [13].

A function $f(z)$ of the form (3), regular in $0<|z|<1$, is said to be meromorphic close-to-convex if there exists a univalent meromorphic starlike function

$$
h(z)=1 / z+b_{0}+b_{1} z+\cdots, \quad 0<|z|<1,
$$

and a real number $\gamma(|\gamma|<\pi / 2)$ such that

$$
\operatorname{Re}\left(z f^{\prime}(z) / h(z) e^{i \gamma}\right)<0, \quad|z|<1,
$$

[6], [12]. We denote this class by $\Gamma$. We define $\Gamma_{0}^{\prime}$ to be the subclass of those $f(z) \in \Gamma$ for which $\gamma=0$ in (6) and $b_{0}=0$ in (5). Equivalently, $f(z)$ belongs to $\Gamma_{0}^{\prime}$ iff there exists a function $\phi(z) \in M C$ such that $\operatorname{Re}\left(f^{\prime}(z) / \phi^{\prime}(z)\right)>0, z \in D$.

Theorem 5. A functional $J\left(f^{\prime}\right)=\operatorname{Re} F\left(f^{\prime}(z), \cdots, f^{(n)}(z), z\right)$ $(0<|z|<1)$ of the type defined for MC attains its maximum and minimum over the class $\Gamma$ only for functions of the form

$$
f^{\prime}(z)=\sum_{j=1}^{N} \chi_{j} \frac{e^{i t_{j}}+z e^{2 i \gamma}}{e^{i t_{j}}-z} \cdot\left(-\frac{1}{z^{2}}\right) \prod_{k=1}^{M}\left(1-z e^{-i \tau_{k}}\right)^{2 m_{k}}
$$

where $N \leqq n+2, \quad M \leqq n+1, \chi_{j} \geqq 0, \quad m_{k} \geqq 0, \sum_{j=1}^{N} \chi_{j}=\sum_{k=1}^{M} m_{k}=1$, and $\sum_{j=1}^{N} \chi_{j}\left(e^{2 i \gamma}+1\right) e^{-i t_{j}}=\sum_{k=1}^{M} 2 m_{k} e^{-i \tau_{k}}$.

The same theorem holds for the class $\Gamma_{0}^{\prime}$ with $\gamma=0$ and the stronger conclusion $\Lambda \leqq n+1$.

A function $f(z)$, regular in $0<|z|<1$, with $f^{\prime}(z)$ defined by (4) is said to be a meromorphic function with boundary rotation $k \pi$ if $m(t)$ is a function of bounded variation satisfying

$$
\int_{0}^{2 \pi} d m(t)=2, \quad \int_{0}^{2 \pi}|d m(t)| \leqq k, \quad \int_{0}^{2 \pi} e^{i t} d m(t)=0 .
$$

We denote this class by $\Lambda_{k}$. Clearly, $M C=\Lambda_{2}$.

TheOREM 6. The functional $J\left(f^{\prime}\right)=\operatorname{Re} F\left(f^{\prime}(z), \cdots, f^{(n)}(z), z\right)$ attains its maximum and minimum over the class $\Lambda_{k}$ only for functions of the form

$$
f^{\prime}(z)=-\frac{1}{z^{2}} \frac{\prod_{j=1}^{M}\left(1-\epsilon_{j} z\right)^{p_{i}}}{\prod_{j=1}^{N}\left(1-e_{j} z\right)^{n_{i}}}
$$


where $M \leqq n+1, N \leqq n+1, \quad\left|\epsilon_{j}\right|=\left|e_{j}\right|=1, \quad p_{j} \geqq 0, \quad n_{j} \geqq 0, \quad \sum_{j=1}^{M} p_{j}$ $-\sum_{j=1}^{N} n_{j}=2, \quad \sum_{j=1}^{M} p_{j} \leqq k / 2+1, \quad \sum_{j=1}^{N} n_{j} \leqq k / 2-1$, and $\sum_{j=1}^{M} p_{j} \epsilon_{j}$
$=\sum_{j=1}^{N} n_{j} e_{j}$.

It is of interest to note that each of the classes $\Lambda_{k}, k>2$, contains some nonunivalent functions. The function $f(z)$ defined by

$$
-z^{2} f^{\prime}(z)=\left(1+2 z \cos \delta+z^{2}\right)^{\alpha} /(1+z)^{\beta}
$$

with $\alpha=(k+2) / 4, \beta=(k-2) / 4,0<\delta<\pi / 2, \cos \delta=\beta / \alpha$ belongs to $\Lambda_{k}$. This function is not univalent if $k>2$ since

$$
-a_{1}=1+2(k-2) /(k+2)>1 \text {. }
$$

4. Concluding remarks. The variational method developed in Theorem 1 can be applied to a wide variety of extremal problems involving various constraints. For example, one might require of the functions in a class that the first $n$ coefficients in the Taylor or Laurent expansions be real, or that some segment of the series have all zero coefficients, e.g. [14] and [7]. The method can also be applied to problems where some fixed coefficient is held constant and acts as an additional parameter in the definition of a class, cf. [1], [2] and $[3]$.

\section{REFERENCES}

1. A. Bielecki, J. Krzyż and Z. Lewandowski, On typically-real functions with a preassigned second coefficient, Bull. Acad. Polon. Sci. Sér. Sci. Math. Astronom. Phys. 10 (1962), 205-208. 799.

2. W. E. Chase, p-close-to-convex functions, Notices Amer. Math. Soc. 15 (1968),

3. M. Finkelstein, Growth estimates of convex functions, Proc. Amer. Math. Soc. 18 (1967), 412-418.

4. S. A. Gelfer, $A$ variational method in the theory of functions of bounded characteristic, Mat. Sb. 69 (111) (1966), 422-433; English transl., Amer. Math. Soc. Transl. (2) 72 (1968), 51-64.

5. G. M. Goluzin, On a variational method in the theory of analytic functions, Leningrad. Gos. Univ. Ucen. Zap. Ser. Mat. Nauk. 23 (1952), 85-101; English transl., Amer. Math. Soc. Transl. (2) 18 (1961), 1-14.

6. R. J. Libera and M. S. Robertson, Meromorphic close-to-convex functions, Michigan Math. J. 8 (1961), 167-175.

7. T. H. MacGregor, Coefficient estimates for starlike mappings, Michigan Math. J. 10 (1963), 277-281.

8. J. A. Pfaltzgraff and B. Pinchuk, $A$ variational method for classes of meromorphic functions (to appear).

9. B. Pinchuk, Extremal problems in the class of close-to-convex functions, Trans. Amer. Math. Soc. 129 (1967), 466-478. 
10. - A variational method for functions of bounded boundary rotation, Trans. Amer. Math. Soc. 138 (1969), 133-144.

11. G. Pólya and M. Schiffer, Sur la representation conforme de l'extérieur d'une courbe fermée convexe, C. R. Acad. Sci. Paris Ser. 248 (1959), 2837-2839.

12. Ch. Pommerenke, Über einige Klassen meromorpher schlichter Funktionen, Math. 7. 78 (1962), 263-284.

13. W. C. Royster, Convex meromorphic functions, MacIntyre Memorial Volume, Ohio University, Athens, Ohio, 1969.

14. M. Schiffer, Univalent functions whose $n$ first coefficients are real, J. Analyse Math. 18 (1967), 329-349.

University of North Carolina at Chapel Hill, Chapel Hill, North

CAROLINA 27514 AND

Princeton University, Princeton, New Jersey 08540

\title{
MAXIMAL FUNCTIONS FOR A CLASS OF LOCALLY COMPACT NONCOMPACT GROUPS
}

\author{
BY KEITH PHILLIPS
}

Communicated by Saunders Mac Lane, September 19, 1968

In this note, we briefly describe some maximal theorem results to be proved in detail in an appendix ( $\$ 4)$ to the paper [PT]. In [PT], maximal averages taken over sets of unbounded measure for functions of several variables over a local field are used to study singular integrals. The results on maximal functions can, however, be obtained for a large class of topological groups, and it is these results which we will describe. The results generalize theorems on maximal functions appearing in $[\mathrm{EH}]$, where the sets over which averages are taken have bounded measures. Let $Z$ denote the integers. Our hypothesis is that $G$ is a locally compact group (written multiplicatively) with left Haar measure $\lambda$ and that $\left\{U_{n}: n \in Z\right\}$ is a neighborhood base at the identity $e$ consisting of relatively compact Borel sets satisfying

(i) $U_{n+1} \subset U_{n}$ for all $n \in Z$ and $\lim _{n \rightarrow-\infty} \lambda\left(U_{n}\right)=\infty$;

(ii) $\lambda\left(U_{n} U_{n}^{-1}\right)<C \lambda\left(U_{n}\right), C$ constant, $n \in Z$;

(iii) For each $n \in Z$ there is an $l(n) \in Z$ such that $U_{l(n)} \supset U_{n}^{-1} U_{n}$ and $U_{j} D U_{n}^{-1} U_{n}$ if $j>l(n)$. And, there is a constant $\alpha$ such that $\lambda\left(U_{l(n)}\right)<\alpha \lambda\left(U_{n}\right)$ for all $n \in Z$.

For such an " $M$-sequence," we can prove [PT] the following theorem. 